\title{
Convection and Rapid Filamentation in Typhoon Sinlaku during TCS-08/T-PARC
}

\author{
HUNG-CHI KUO* \\ Department of Atmospheric Sciences, National Taiwan University, and Taida Institute \\ for Mathematical Sciences, Taipei, Taiwan \\ ChIH-Pei Chang \\ Department of Meteorology, Naval Postgraduate School, Monterey, California, and Department of \\ Atmospheric Sciences, National Taiwan University, Taipei, Taiwan \\ CHING-HwANG LIU* \\ Department of Atmospheric Sciences, Chinese Culture University, Taipei, Taiwan
}

(Manuscript received 24 September 2010, in final form 17 February 2012)

\begin{abstract}
This study examines the convection and rapid filamentation in Typhoon Sinlaku (2008) using the Naval Research Laboratory (NRL) P-3 aircraft data collected during the Tropical Cyclone Structure 2008 (TCS-08) and The Observing System Research and Predictability Experiment (THORPEX) Pacific Asian Regional Campaign (T-PARC) field experiments. The high-resolution aircraft radar and wind data are used to directly compute the filamentation time, to allow an investigation into the effect of filamentation on convection. During the reintensification stage, some regions of deep convection near the eyewall are found in the vorticity-dominated area where there is little filamentation. In some other parts of the eyewall and the outer spiral rainband region, including areas of upward motion, the filamentation process appears to suppress deep convection. However, the magnitude of the suppression differs greatly in the two regions. In the outer spiral band region, which is about $200 \mathrm{~km}$ from the center, the suppression is much more effective, such that the ratio of the deep convective regime occurrence over the stratiform regime varies from around 50\% (200\%) for filamentation time shorter (longer) than $24 \mathrm{~min}$. In the eyewall cloud region where the conditions are conducive to deep convection, the filamentation effect may be quite limited. While effect of filamentation suppression is only about $10 \%$, it is still systematic and conspicuous for filamentation times shorter than $19 \mathrm{~min}$. The results suggest the possible importance of vortex-scale filamentation dynamics in suppressing deep convection and organizing spiral bands, which may affect the development and evolution of tropical cyclones.
\end{abstract}

\section{Introduction}

In general turbulence dynamics, convective systems have a tendency for a downscale cascade of energy, as energy is transferred to the smallest scale and dissipated.

\footnotetext{
* Additional affiliation: Department of Meteorology, Naval Postgraduate School, Monterey, California.

Corresponding author address: Prof. Hung-Chi Kuo, Department of Atmospheric Sciences, National Taiwan University. No. 1, Sec. 4, Roosevelt Road, Taipei, 10617 Taiwan.

E-mail:kuo@as.ntu.edu.tw
}

However, turbulence in a rotating fluid can be quite different as upscale transfer of energy may occur in the presence of rotation (e.g., Matthaeus and Montgomery 1980; McWilliams 1984). In a strong rotating environment with a random field of vorticity, coherent vortices may emerge by axisymmetrization and move around under each other's far-field circulation. They can merge when two similar vortices move close enough to each other (e.g., Dritschel and Waugh 1992; Kuo et al. 2008). As a result, the vortices on the average become fewer and larger with time. The vorticity-generating processes from the convective systems and the upscale transfer of energy and merger dynamics in the cyclone environment 
may play a pivotal role in the genesis and intensification of tropical cyclones (TCs). The vorticity field generated by the convective systems in the cyclone environment is a critical mechanism for increasing the TC circulation. The TC vortex provides the horizontal shear that can organize convection toward banded structures, with the characteristic time of the band formation determined by the strain effect of the cyclone. In addition, the horizontal shear deformation may enhance the cloud entrainment and mixing if the characteristic time of the filamentation process induced by the strain effect is comparable to the convective overturning time. This enhanced entrainment will suppress convection in the cyclone environment.

Rozoff et al. (2006, hereafter R06) defined a local parameter, the "filamentation time," which is the $e$-folding time for the growth of the vorticity gradient. They used this parameter to propose the idea of a rapid filamentation zone (RFZ) outside the radius of maximum wind (RMW) of TCs. The RFZ is a region of horizontal strain-dominated flow where the filamentation time is shorter than the approximate 30 -min moist convective overturning time. Deep convection in the RFZ may become highly distorted and even suppressed, leading to the formation of a moat. Through a fully compressible, three-dimensional, nonhydrostatic model simulation, Wang (2008) found that the rapid filamentation $(\mathrm{RF})$ process can effectively suppress high-azimuthal wavenumber asymmetries immediately outside the RMW, but has little effect on well-organized low wavenumber spiral bands. He argued that the RF may play a secondary role in the formation of the moat in TCs. However, when a TC becomes very strong, it is possible that the subsidence is confined to the edge of the deep convection and strengthened by inertial stability (e.g., Rozoff et al. 2008). When a moat region is of a sufficiently large size in a TC with concentric eyewalls, the impact of subsidence is unlikely to be uniform throughout the moat, which leaves the possibility that the RF dynamics may contribute to the organization of the moat. This is more likely to happen in the western North Pacific due to the presence of very intense typhoons and largeradius concentric eyewall typhoon cases (Hawkins et al. 2006). Kuo et al. (2009) showed that the RF process can be important in the organization of the moat in strong typhoons in the western North Pacific, at least in cases with maximum winds greater than $130 \mathrm{kt}$ that developed concentric eyewalls.

Past studies of the filamentation dynamics and the influence on convection have been based on theories, numerical modeling, and/or satellite observations. Direct analysis of the effects of filamentation dynamics on the development of convection has been lacking, especially in a TC environment, because of the lack of high-resolution wind data. The Tropical Cyclone Structure 2008 (TCS-08) and The Observing System Research and Predictability Experiment (THORPEX) Pacific Asian Regional Campaign (T-PARC) field experiments, conducted during August-October in 2008, were the most intensive field observational programs carried out over the tropical western North Pacific (Elsberry and Harr 2008). The high-resolution winds retrieved from the Naval Research Laboratory (NRL) P-3 airborne radar in TCS-08/T-PARC allow a direct computation of the filamentation time in typhoons. Coupled with radar observations, it becomes possible to investigate the effect of filamentation on convection. The purpose of this work is to study the impact of the filamentation dynamics on convection during the reintensification stage of Typhoon Sinlaku, including both its eyewall and outer rainband regions. Section 2 describes the data and the analysis method. Section 3 discusses the relationship between convection and filamentation dynamics, and section 4 is a summary.

\section{Data and method}

According to the Joint Typhoon Warning Center (JTWC) analysis, Sinlaku (WP15, 2008) became a named typhoon at 0000 UTC 9 September 2008 (Fig. 1). On 1800 UTC 10 September, it reached maximum intensity with winds up to $125 \mathrm{kt}\left(1 \mathrm{kt}=0.5144 \mathrm{~m} \mathrm{~s}^{-1}\right)$ and minimum central sea level pressure of $929 \mathrm{hPa}$. It then slowly weakened and its maximum wind speed decreased to $35 \mathrm{kt}$ on 0000 UTC 17 September. After the weakening phase, it began its second phase of intensification, in which the maximum wind increased to $70 \mathrm{kt}$ and minimum pressure decreased to $970 \mathrm{hPa}$ on 0600 UTC 19 September. During the reintensification of Sinlaku, four aircraft missions involving both the NRL P3 and the Air Force Reserve WC130 were flown. In the second mission the NRL P-3 took off from Yokoda Air Base in Japan at 2230 UTC 17 September and flew near the eyewall of Typhoon Sinlaku at 0200 UTC 18 September at an altitude of $3100 \mathrm{~m}$, which is optimal for the utilization of the onboard Electra Doppler Radar (ELDORA; Figs. 1 and 2). The NRL P-3 first approached the eyewall from the eastern side of Sinlaku, then circled counterclockwise to the northern and western sectors. The blue line in the bottom-right panel in Fig. 1 indicates the time of the NRL P-3 observations with respect to the variation of maximum wind speed and minimum sea level pressure.

Figure 2 is the Moderate Resolution Imaging Spectroradiometer (MODIS) Terra visible satellite picture overlapped with the NRL P-3 flight track. During the 


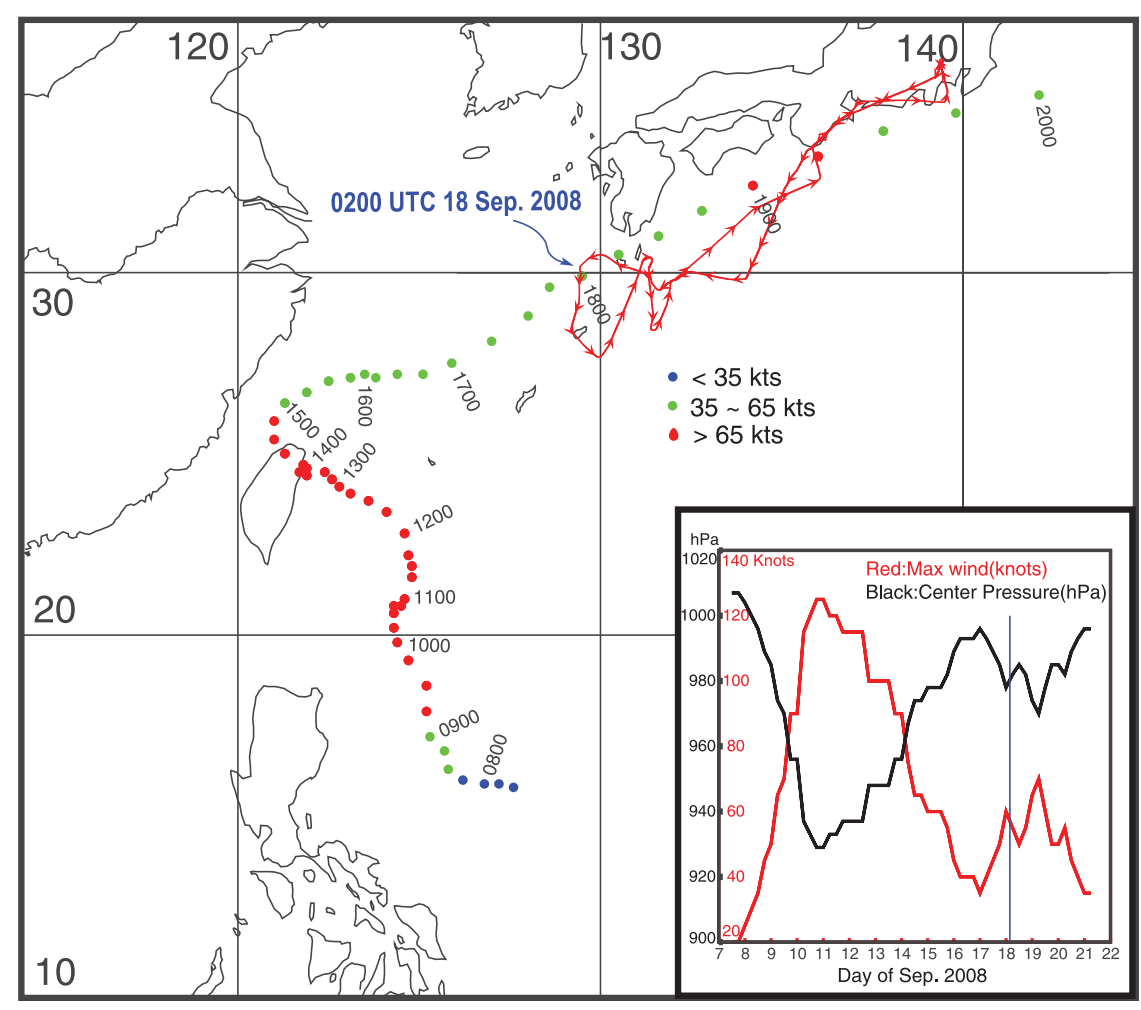

FIG. 1. Typhoon Sinlaku (2008) track (dots) and NRL P-3 flight (red line with arrow head indicating the airplane's direction). The maximum wind speed higher than $65 \mathrm{kt}$, between 65 and $35 \mathrm{kt}$, and lower than $35 \mathrm{kt}$ are indicated in red, green, and blue, respectively. (bottom right) The typhoon maximum wind speed (red line, $\mathrm{kt}$ ) and center pressure (black line, hPa) are shown. The blue line indicates the time when the NRL P-3 flew near the eyewall.

flight time, the principal rainband was about $200 \mathrm{~km}$ east of the TC center. The vertical profile of temperature and dewpoint temperature from dropsondes $4,6,8$, and 11 are shown in Fig. 3. These soundings are chosen to study the different atmospheric structures between the convective regions associated with the TC center (4, region A) and the principal rainband (11, region $B)$ and the region between them. Sounding 4 was not saturated in the lower troposphere, which indicates that convection in the eyewall region had gaps between convective updrafts and that mesoscale saturation did occur. Sounding 4 also suggests the presence of subsidence, which may be associated with the overturning flow from eyewall convection. The presence of subsidence in the low convective region between regions $\mathrm{A}$ and $\mathrm{B}$ is evident in soundings 6 and 8 , which matches the clear region defined in the satellite imagery of Fig. 2.

The NRL P-3 aircraft was equipped with the fore and aft X-band ELDORA radar (Hildebrand et al. 1996). The fore/aft scanning geometry is such that sweeps of the scans are approximately $20^{\circ}$ normal to the fuselage, while the antennas rotate about an axis parallel to the longitudinal axis of the aircraft. As a result, the along-track spatial resolution (i.e., the distance between two consecutive fore or aft scans) is about $1 \mathrm{~km}$. Using the dualDoppler radar synthesis technique, the horizontal wind field can be derived from the radial velocities at beam intersections (Wakimoto et al. 1998). The radar data were carefully edited by removing the sea clutter and second trip echo using the National Center for Atmospheric Research (NCAR) Solo II radar software. Using the NCAR REORDER radar interpolation software, the radial velocity and reflectivity were interpolated onto threedimensional Cartesian grids with grid spacing of $0.5 \mathrm{~km}$ in horizontal and vertical. The REORDER software usually provides good results using the "CRESSMAN" (Cressman 1959) weighting function when the flight track is straight. If the track is curved, the "closest point" weighting function will be used. Finally, the dual-Doppler winds were obtained using the NCAR CEDRIC synthesis software with the Leise two-step filter (Leise 1982) applied. We have also calculated the dual-Doppler winds based on the variational interpolation scheme proposed by Gamache (1997). This method uses many constraints to solve for the dual-Doppler winds and usually provides reasonable results for the curved 


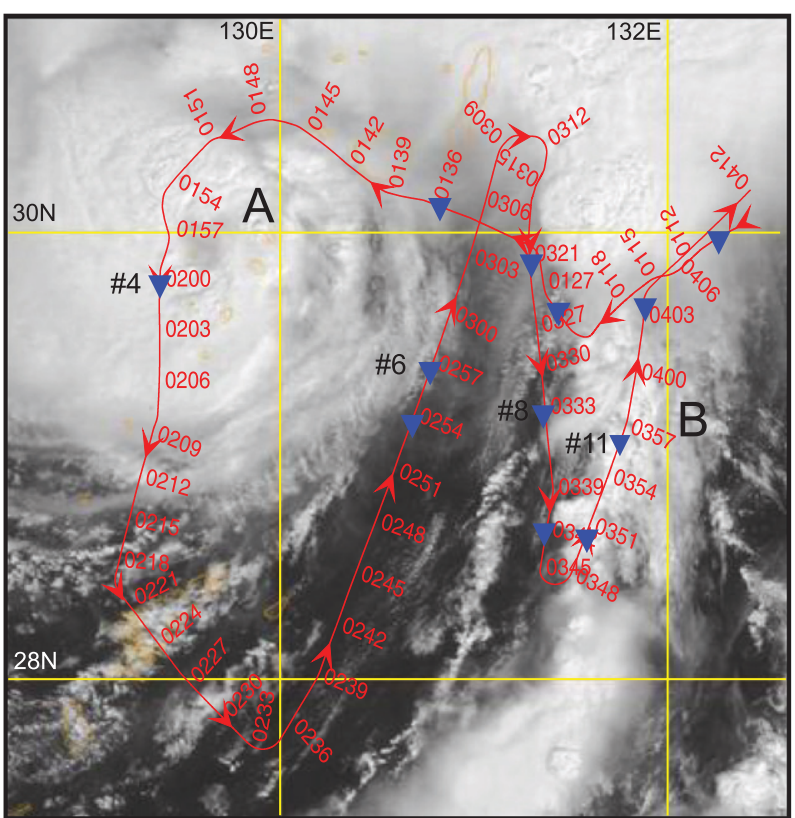

FIG. 2. The NRL P-3 flight superimposed on 0141 UTC 18 Sep 2008 MODIS Terra satellite visible image. The red line is the aircraft flight track with hour and minute labeled (UTC). The red arrow heads indicate the aircraft's direction. The blue triangles indicate the dropsonde locations. The 4, 6, 8, and 11 dropsondes are analyzed in this paper. Letters A and B indicate the regions for our dualDoppler analysis during the periods 0139-0205 and 0352-0403 UTC 18 Sep 2008, respectively.

flight track. We have compared the winds from both methods and the results are similar.

The aircraft radar can thus provide a nearly real-time three-dimensional wind field up to 50-km range from the aircraft along the flight track. These two radars also collect three-dimensional reflectivity at very high spatial resolution (approximately $300 \mathrm{~m}$ ), which can be used to study the characteristics of convection in the TC environment.

The filamentation time is the $e$-folding time scale for growth of the vorticity gradient and was defined by R06:

$$
\tau_{\text {fil }}=2 / \sqrt{S_{1}^{2}+S_{2}^{2}-\zeta^{2}}, \quad \text { if } \quad S_{1}^{2}+S_{2}^{2}-\zeta^{2}>0,
$$

where $S_{1}=\partial u / \partial x-\partial v / \partial y$ and $S_{2}=\partial v / \partial x+\partial u / \partial y$ are the rates of strain, and $\zeta=\partial v / \partial x-\partial u / \partial y$ is the relative vorticity. In the literature, $1 / 2 S_{1}$ is referred to as the stretching deformation, $1 / 2 S_{2}$ as the shearing deformation, and $1 / 2\left(S_{1}^{2}+S_{2}^{2}\right)^{1 / 2}$ as the total deformation. Note that the filamentation time calculations involve the assumption of slow evolving velocity gradient with respect to the vorticity gradient following a fluid parcel. In other words, if the velocity gradient changes faster than the filamentation time, the flow may change appreciably before the expected filamentation takes effect. The diagnosis in such a situation will be meaningless. Using the dual-Doppler wind, the filamentation time may be computed at every data point. The RFZ has been defined to coincide with a $\tau_{\text {fil }}<30 \mathrm{~min}$ based on an estimate of 30-min convective overturning time (R06). However, in this study it will be set at $\tau_{\text {fil }}<25 \mathrm{~min}$ in view of the more vigorous convection in a TC environment. It appears that the storm-scale wind in general will not change significantly within an hour, and the filamentation time diagnosis concept is applicable. We also define the slow filamentation zone (SFZ) for areas with $\tau_{\text {fil }}>25 \mathrm{~min}$ and the vorticity dominated zone (VDZ; $\left.S_{1}^{2}+S_{2}^{2}-\zeta^{2}<0\right)$ as areas in which no filamentation time can be calculated.

We classify the clouds in Typhoon Sinlaku (2008) into convective and stratiform types by adapting the method of Steiner et al. (1995) with modifications that account for the difference between different types of radars. The classification of deep cumulus convection according to Steiner et al. (1995) is simple and straightforward: if the reflectivity is greater than $40 \mathrm{dBZ}$, or the difference between a grid point and its $11-\mathrm{km}$ background is greater than certain threshold, this grid point is classified as deep cumulus convection. Any reflectivity less than $10 \mathrm{~dB} Z$ is not counted as deep cumulus convection. Accordingly, all the nonconvective grid points are classified as stratiform cloud. Neither the horizontal gradient nor the vertical structure of the reflectivity has been considered in the method. An improvement of this method was proposed by Biggerstaff and Listemaa (2000), in which several reflectivity structure details, including the horizontal and vertical gradients and brightband characteristics (Rosenfeld et al. 1995), are incorporated into the scheme. The data used in these studies are from the Next Generation Weather Radar (NEXRAD) radar, which have a coarser resolution in range and more limited elevations in the vertical direction compare to the ELDORA radar used in TCS08. On the other hand, the ELDORA radar, as a X-band radar, suffers more attenuation than the S-band type NEXRAD. To apply the convection classification scheme to the ELDORA radar data, some parameter adjustments are required. Comparing the reflectivity between S-band and X-band radar measurement under heavy rainfall regime, Anagnostou et al. (2006) have shown that the reflectivity difference between S-Pol and X-Pol at $25 \mathrm{~km}$ is about $10 \mathrm{dBZ}$, and about $20 \mathrm{dBZ}$ at $40 \mathrm{~km}$. To account for the attenuation of $\mathrm{X}$-band radar, we relax the convective reflectivity threshold from 40 to $30 \mathrm{dBZ}$. Our maximum threedimensional radius of influence is about $2.2 \mathrm{~km}$ for interpolating the radar data onto the grid points, which 


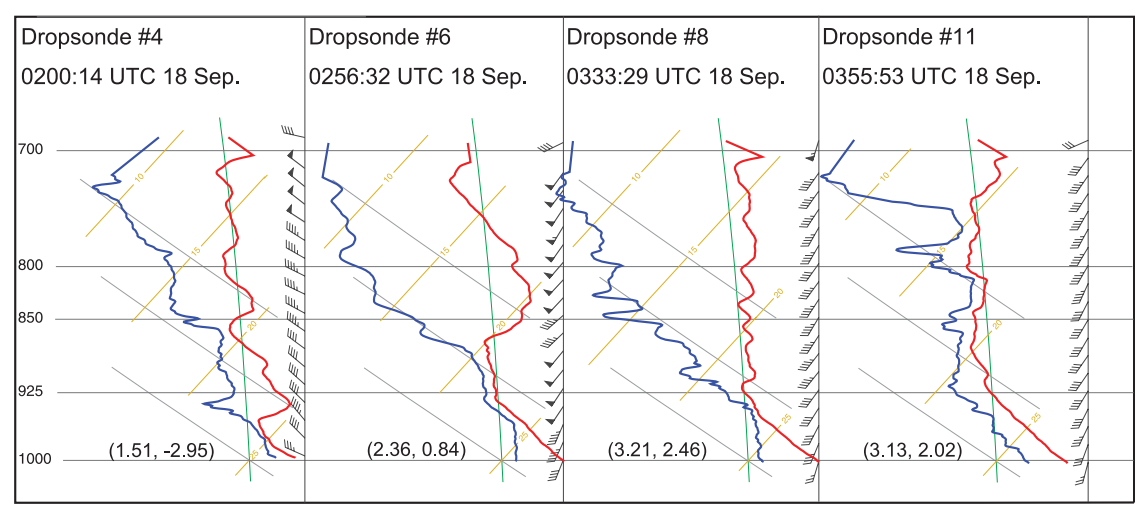

FIG. 3. NRL P-3 dropsondes 4, 6, 8, and 11 on 18 Sep 2008. The release times are indicated at the top and the vertical wind shear $\left(10^{3} \mathrm{~s}^{-1}\right)$ of east-west and north-south components between $3000 \mathrm{~m}$ and surface are indicated at the bottom. Red lines are temperature and blue lines are the dewpoint temperature. The green line indicates the $25^{\circ} \mathrm{C}$ moist adiabat. Dropsonde winds are plotted on the right-hand side of each. Half barb is $2.5 \mathrm{~m} \mathrm{~s}^{-1}$, one barb is $5 \mathrm{~m} \mathrm{~s}^{-1}$, and black triangle is $25 \mathrm{~m} \mathrm{~s}^{-1}$.

gives a background reflectivity range of approximately $9 \mathrm{~km}$ with the two-step Leise filter. We have also tested the background reflectivity range of 5,7, and $11 \mathrm{~km}$ and have seen similar results. Thus, we use the convective threshold of $30 \mathrm{dBZ}$ and the background reflectivity range of $9 \mathrm{~km}$, instead of $40 \mathrm{dBZ}$ and $11 \mathrm{~km}$, respectively, used by Steiner et al. (1995). The convection types are determined from the "working level" $[1.5-\mathrm{km}$ height near the radar and 3-km height far away from radar in Steiner et al. (1995)]. The working level is chosen below the bright band to avoid the brightband contamination, and also not too close to the sea surface to avoid the ground clutter contamination. We have found in our radar data that the melting level is consistently at about $5-\mathrm{km}$ height. Therefore, we chose the midlevel $2.5 \mathrm{~km}$ between the surface and bright band as our working level.

\section{Filamentation dynamics and convection}

Figure 4a presents the storm-relative dual-Doppler wind (black bold vector) superimposed on the reflectivity at $2 \mathrm{~km}$ above the mean sea level. The RFZs $\left(\tau_{\text {fil }}<\right.$ $25 \mathrm{~min}$ ) are hatched in blue color, and the white bold contour indicates the convective area. Over the aircraft path around the outer portion of the eye, the reflectivity pattern at $2 \mathrm{~km}$ (Fig. 4a) defines several convective rainbands that wrap into the center. Figure 4 a clearly indicates that deep convection covers only a limited area in the typhoon core region, and most of the RFZ overlaps with the stratiform area inside the convective spiral bands.

Figure $4 \mathrm{~b}$ shows the RFZs and VDZs in yellow and light green colors, respectively. The blank area is the
SFZs. The convective bands, as shown in Fig. 4a, are in gray shaded area with blue outlines. The irregular shapes of the high-vorticity areas could be due to vortex Rossby waves (Montgomery and Kallenbach 1997). An RFZ is found around $20-40 \mathrm{~km}$ from the TC center, which is immediately outside the high-vorticity region approximately $10-20 \mathrm{~km}$ from the center. The RFZ is also clearly asymmetric. The asymmetry of the RFZ may be in part due to the asymmetry of the vorticity areas near the eyewall region, as the filamentation time may be lengthened by the presence of the vorticity. In general, the high-vorticity region and this RFZ are separated by the RMW at approximately $20 \mathrm{~km}$ from the center.

There are two convective bands that wrap into the center from the northeastern quadrant. The inner convective band near the TC center is mostly collocated with a VDZ about $15 \mathrm{~km}$ from the center. The outer convective band that wrapped into the center from the west encounters a RFZ in the northwest quadrant about $20 \mathrm{~km}$ from the center. In the eyewall region at $2-\mathrm{km}$ altitude, the VDZ occupies approximately $40 \%$ of the area and RFZ about $35 \%$ of the area. The overlapping of deep convection and vorticity may increase the heating efficiency and enhance the conversion of potential to kinetic energy (Hack and Schubert 1986), which may be expected in the reintensification stage of Typhoon Sinlaku.

Figure $4 \mathrm{c}$ is the same as Fig. $4 \mathrm{~b}$ except for upward $\left(>0.5 \mathrm{~m} \mathrm{~s}^{-1}\right.$, shaded in yellow) and downward $(<-0.5$ $\mathrm{m} \mathrm{s}^{-1}$, shaded in green) motions. Figure $4 \mathrm{c}$ indicates general areas of subsidence between $10-25-\mathrm{km}$ radius from the center and also the presence of several small areas of updrafts. Figure 5 is the same as Fig. 4 except 

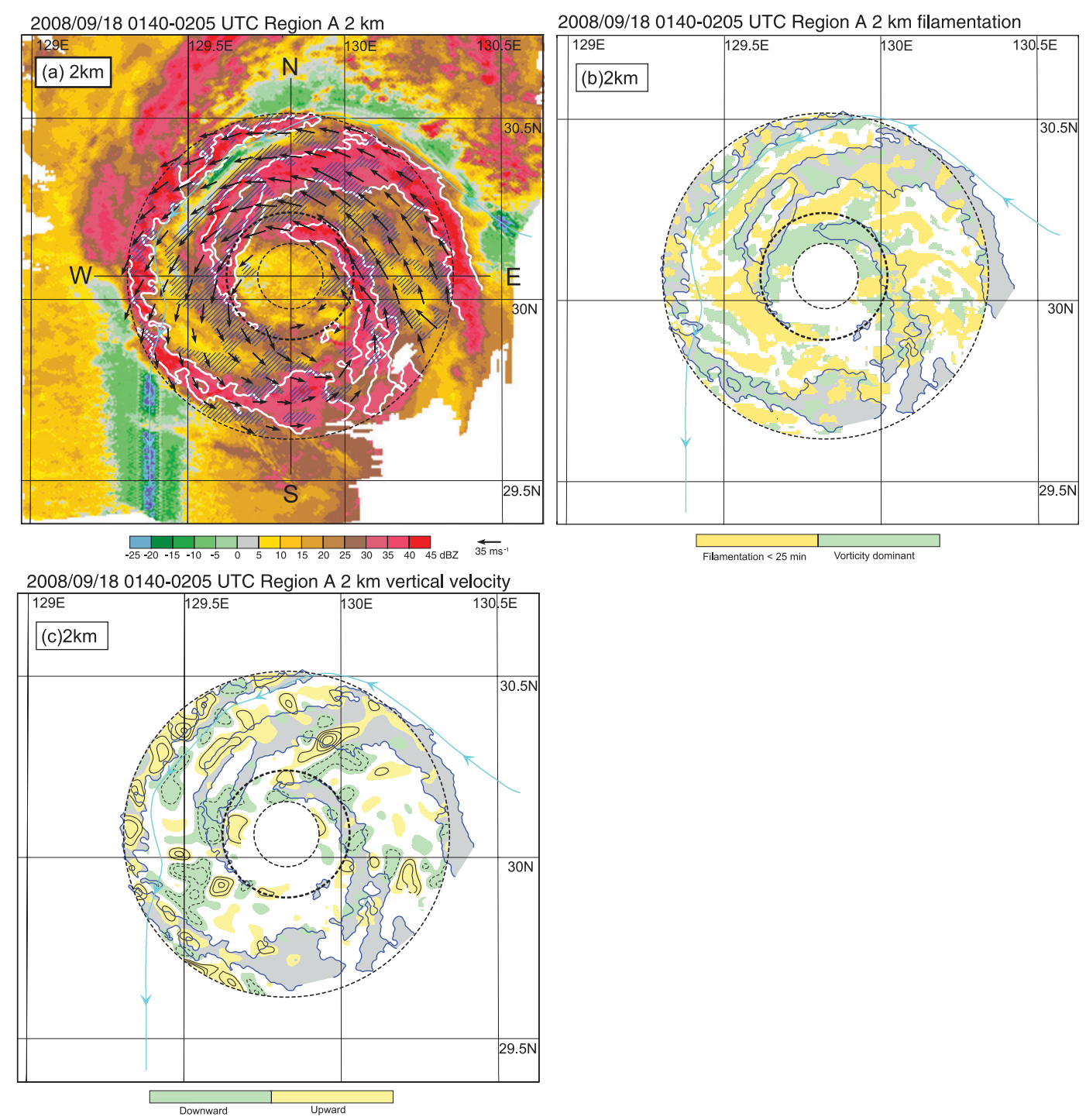

FIG. 4. (a) 0140-0205 UTC 18 Sep 2008 NRL P-3 storm-relative dual-Doppler wind (black bold vector with scale indicated at the bottom-right corner) superimposed on the ELDORA radar reflectivity (dBZ scale at the bottom) at $2 \mathrm{~km}$ above sea level. The thin solid black lines are the latitude and longitude at every $0.5^{\circ}$. Two cross-sectional $(\mathrm{E}-\mathrm{W}, \mathrm{N}-\mathrm{S})$ locations are also indicated in thick black lines. The light blue line is the P-3 flight track and the arrow heads indicate the aircraft heading direction. The bold white contour indicates the convective area and the RFZs are hatched in blue. The thin dashed circles indicate the distance of 10 and $50 \mathrm{~km}$ from the typhoon center, the thick dashed circle is the radius of maximum wind. (b) The RFZs and VDZs are in yellow and light green colors, respectively. The gray shaded area with blue outline is the convective area. (c) As in (b), but for upward $\left(>0.5 \mathrm{~m} \mathrm{~s}^{-1}\right.$ shaded in yellow) and downward $\left(<-0.5 \mathrm{~m} \mathrm{~s}^{-1}\right.$ shaded in green) motions. The contour interval is $1 \mathrm{~m} \mathrm{~s}^{-1}$.

for 4-km altitude. The RFZ, SFZ, and VDZ features are generally similar between the two levels with the updraft stronger at $4 \mathrm{~km}$. In general, the updrafts are located 5-10 km inside the RMW. This is in agreement with the results of Jorgensen (1984), who showed that the maximum convective-scale updrafts are typically located between 1 and $6 \mathrm{~km}$ inward from the RMW. Note that the convective band in the east about $20 \mathrm{~km}$ from the center is mostly collocated with RFZ (VDZ) at 2-km $(4 \mathrm{~km})$ height. There are several areas of strong updrafts in the east convective band at 4-km height. The vertical motion figures also reveal that the convective area in the west about $25 \mathrm{~km}$ from the center is mostly under the influence of downward motion, and some small areas of low reflectivity stratiform area are under the influence of upward motion. This suggests that the effects of filamentation and vertical motion may either reinforce or resist each other. 


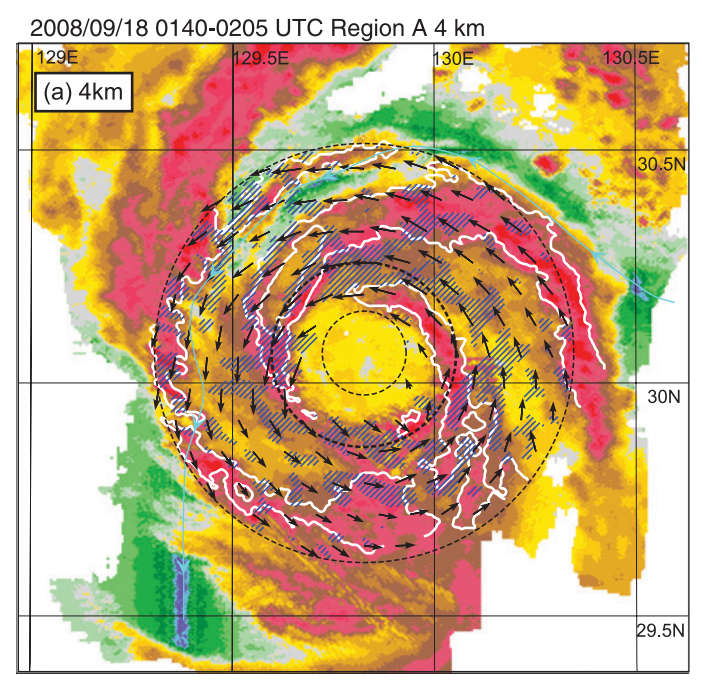

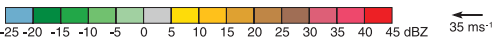
2008/09/18 0140-0205 UTC Region A 4 km vertical velocity

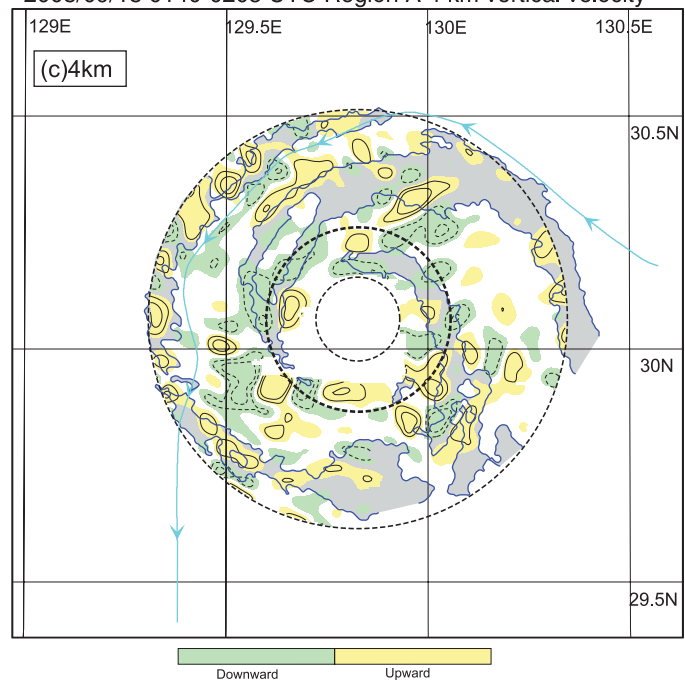

Figure 6 shows the east-west (Fig. 6a) and northsouth (Fig. 6b) cross sections through the typhoon center. The white bold bars at the bottom of the figures indicate the convective areas. Also plotted is the RFZ in the blue hatched area. The high reflectivity that extends to $8-\mathrm{km}$ height at about $20 \mathrm{~km}$ from the center characterizes the inner core of the eyewall (Fig. 6a). A stratiform area is located on the southern side of the typhoon (Fig. 6b) with a 20-dBZ echo top at around 5-km height. Note that the west (east) eyewall is dominated by downward (upward) motion. The asymmetry of the vertical motion may be due to the fact that the typhoon is in a westerly vertical shear, so the upward motion is enhanced on the downshear side (Zhu et al. 2004). Figure 6 reveals that RFZs are mostly collocated with the stratiform area and overlapped with some convective area.
2008/09/18 0140-0205 UTC Region A 4 km filamentation

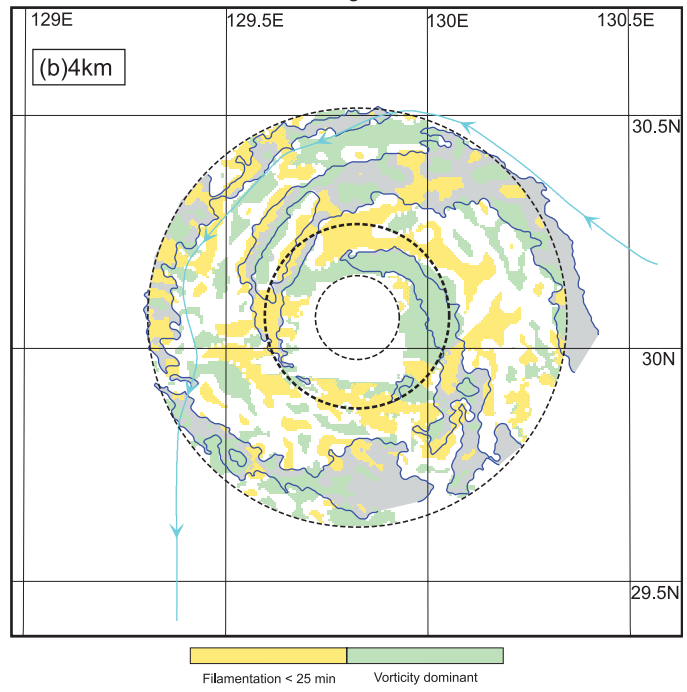

FIG. 5. As in Fig. 4, but at $4 \mathrm{~km}$, and that in (c) vertical motion $>1 \mathrm{~m} \mathrm{~s}^{-1}$ and $<-1 \mathrm{~m} \mathrm{~s}^{-1}$ are shaded and the contours interval is $2 \mathrm{~m} \mathrm{~s}^{-1}$.

Examination of region B (outer spiral band) at 2-km altitude reveals that the RFZ (Fig. 7a) is mostly collocated with the stratiform area and downward motion region in the inner side of the rainband. Didlake and Houze (2009) reported that a convective-free region is often observed in the TC intensifying stage between the eye core and the principal rainband. They suggested that the vorticity dynamics of the overturning updraft and inner-edge downdraft may sustain the principal rainband and help to make it effectively stationary relative to the storm center. The inner-edge downdraft, with the evaporative cooling enhancement, may create a sharp reflectivity gradient along the inner boundary of the rainband. While the subsidence contributes to the suppression of convection in the region between the eye core and the rainband, it is possible that filamentation 
2008/09/18 0140-0205 UTC Region A E-W and N-S cross-section
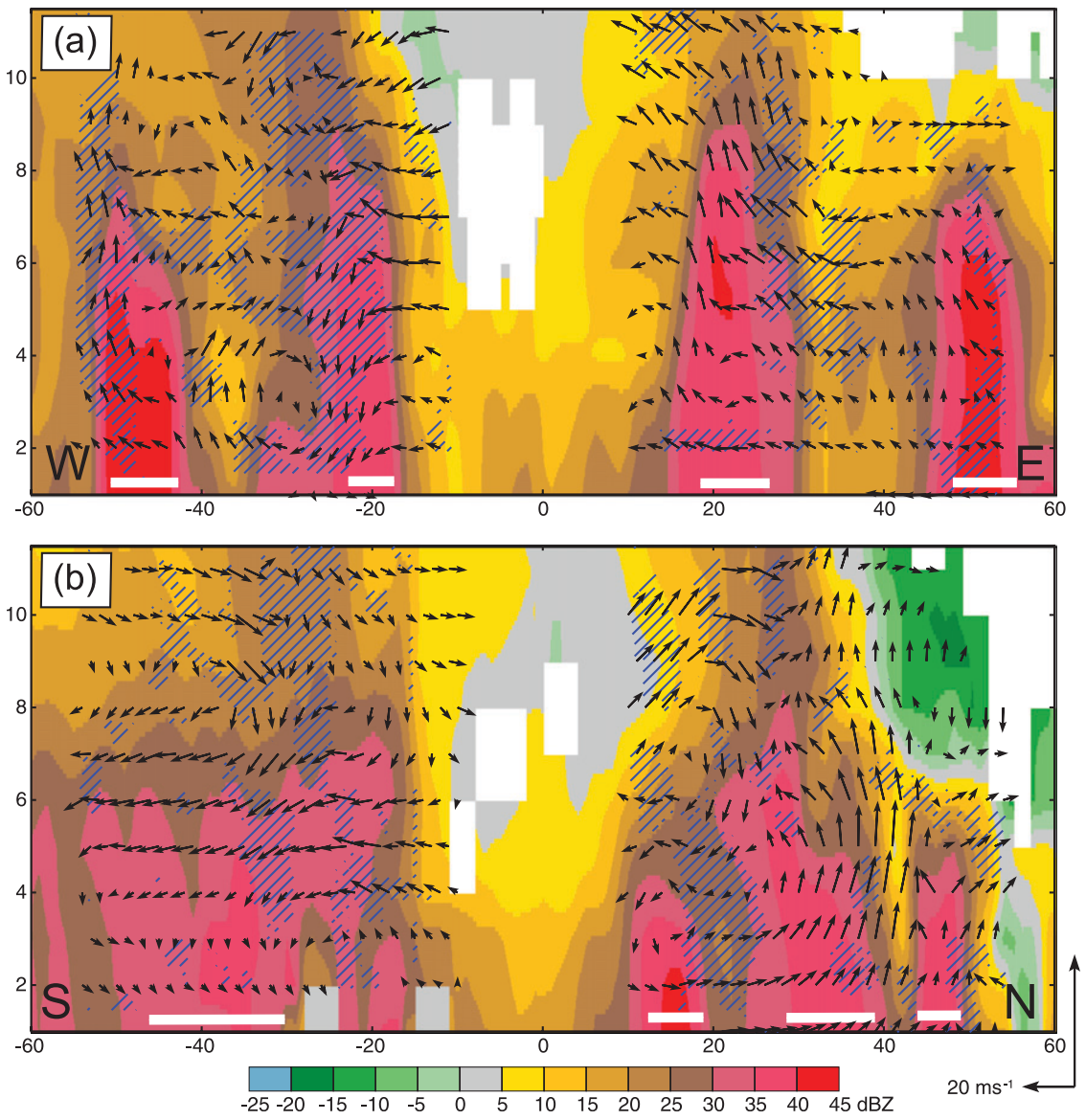

FIG. 6. (a) E-W and (b) N-S vertical cross sections through the typhoon center for region A indicated in Fig. 4a. The color background is the ELDORA radar reflectivity with color scale shown at the bottom. Vectors are the storm-relative cross winds with scale indicated at the bottom-right corner. The blue hatched area are RFZs and the white bold bars at the bottom of the figures indicate convective areas.

dynamics due to the strain effect may also be important. This is evident in the fact that RFZ occurs in the inner side of the Sinlaku's outer rainband (Fig. 7c). Our observation also indicates that the vorticity field in the rainband is coupled with deep convection (Fig. 7b). The vorticity may lengthen the filamentation time, thus offsetting the straining flow and resist the filamentation effect. In other words, the coupling of vorticity contributes to the very survival of deep convection in the relatively hostile environment of strain flow. Figures 4-7 suggest that RFZs exist in both the eyewall and the outer rainband regions during the reintensification stage of Typhoon Sinlaku. The combined processes of RF and subsidence are most relevant to the outer spiral band. These observations are consistent with Wang's (2008) numerical results that RFZ provides a favorable environment for the formation and organization of spiral rainbands.
Figure 8a shows the frequency distribution of the filamentation time (minute) for convective and stratiform reflectivity between 2 and $4 \mathrm{~km}$ in region $\mathrm{A}$. The dashed line in the top panel is the difference between the frequencies of the two. In the eyewall region the frequency of the deep convection peaks sharply at $\tau_{\text {fil }} \sim 20 \mathrm{~min}$ with $3.5 \%$ of the echoes, while that of the stratiform area peaks at $\tau_{\text {fil }} \sim 17.5 \mathrm{~min}$ with $4 \%$ of the echoes. The differences between the peaks of filamentation time of the stratiform and convective areas in region A may not be statistically significant, as the Student's $t$ test gives only a confidence level of $75 \%$. The difference between the two profiles, however, indicates a slight preference of stratiform reflectivity when $\tau_{\text {fil }}$ is shorter. The demarcation occurs at $\tau_{\text {fil }}=19 \mathrm{~min}$. This difference is not large, around $1 \%$ out of $\sim 4.5 \%$ near $\tau_{\text {fil }}=15 \mathrm{~min}$, but is nevertheless systematic when the regions of $\tau_{\text {fil }}>25 \mathrm{~min}$ 

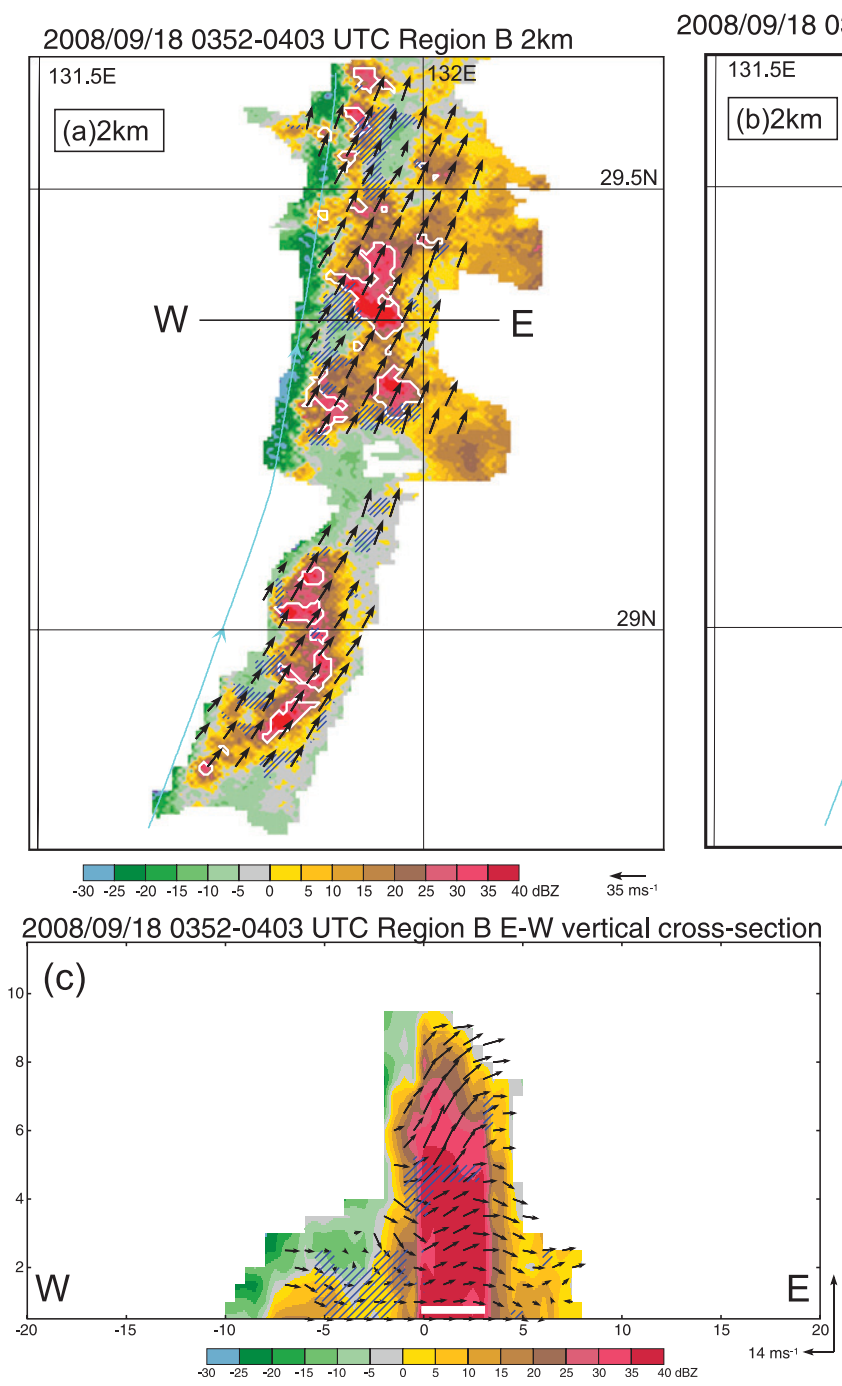

2008/09/18 0352-0403 UTC Region B 2 km filamentation
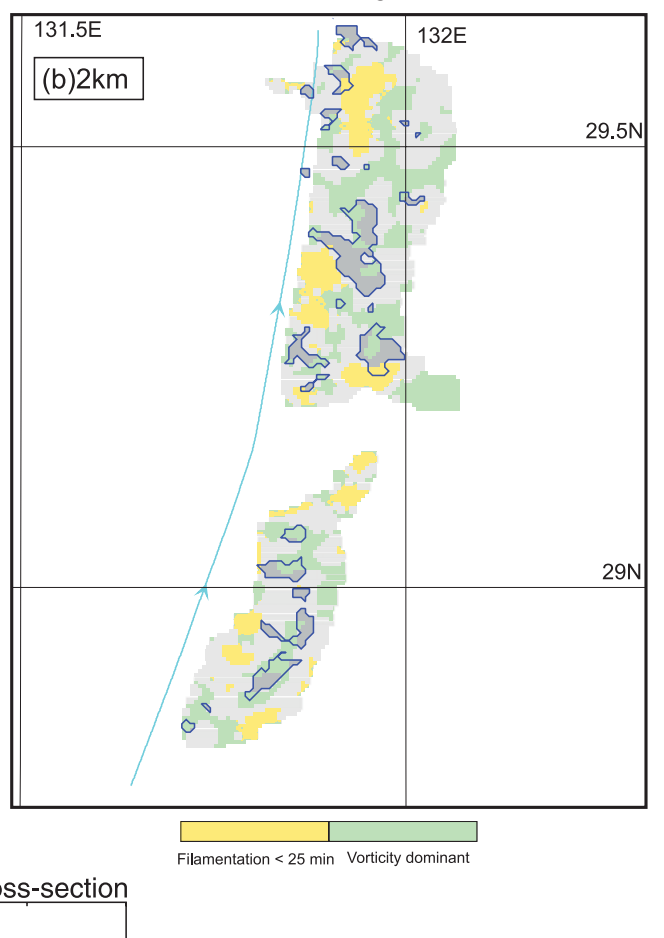

FIG. 7. (a),(b) As in Fig. 4, but for region B from 0352 to 0403 UTC 18 Sep 2008. (c) As in Fig. 6a, but for region B at the location indicated in (a). and $\tau_{\text {fil }}<19 \mathrm{~min}$ are compared. Figure 8 suggests that for a filamentation time shorter than $19 \mathrm{~min}$, the stratiform regime dominates, and for a filamentation time longer than $19 \mathrm{~min}$, the convective regime dominates. This means that even in the eyewall region where vigorous forcing of intensive deep convection can be expected to effectively resist RF, the suppression effect of the filamentation process may still be present.

In the outer spiral band region $200 \mathrm{~km}$ away from the TC center (region B), the evidence of a RF process being associated with stratiform area is much more evident (Fig. 8b). A clear demarcation exists at approximately $24 \mathrm{~min}$, where the fast filamentation regime that suppresses deep convection is separated from the filamentation regime in which deep convection dominates. The suppression of deep convection suggests a reduction of their frequency to as much as $2 \%$ less of the low reflectivity at $\tau_{\text {fil }}=10 \mathrm{~min}$, compared with a $1.5 \%$ higher frequency when $\tau_{\text {fil }}>24 \mathrm{~min}$. The results are statistically significant as the Student's $t$ test in region B gives a confidence level of $98 \%$.

To examine the relationship between filamentation time and the vertical motion for the convective and stratiform regimes, the frequency distribution of filamentation time for convective and stratiform regimes is defined in Fig. 9 for regions of upward motion (top panel) and downward motion (bottom panel) in the eyewall region (region $\mathrm{A}$ ) and the outer rainband region (region B), respectively. The frequency is computed as in Fig. 8.

In the outer rainband region (Fig. 9b), the distribution of stratiform regime is symmetric with respect to the sign of vertical motion, but that of convective regime is asymmetric. The deep convection in the upward motion regime is $2.5-3$ times more frequent than in the downward motion regime. This difference in symmetry may 

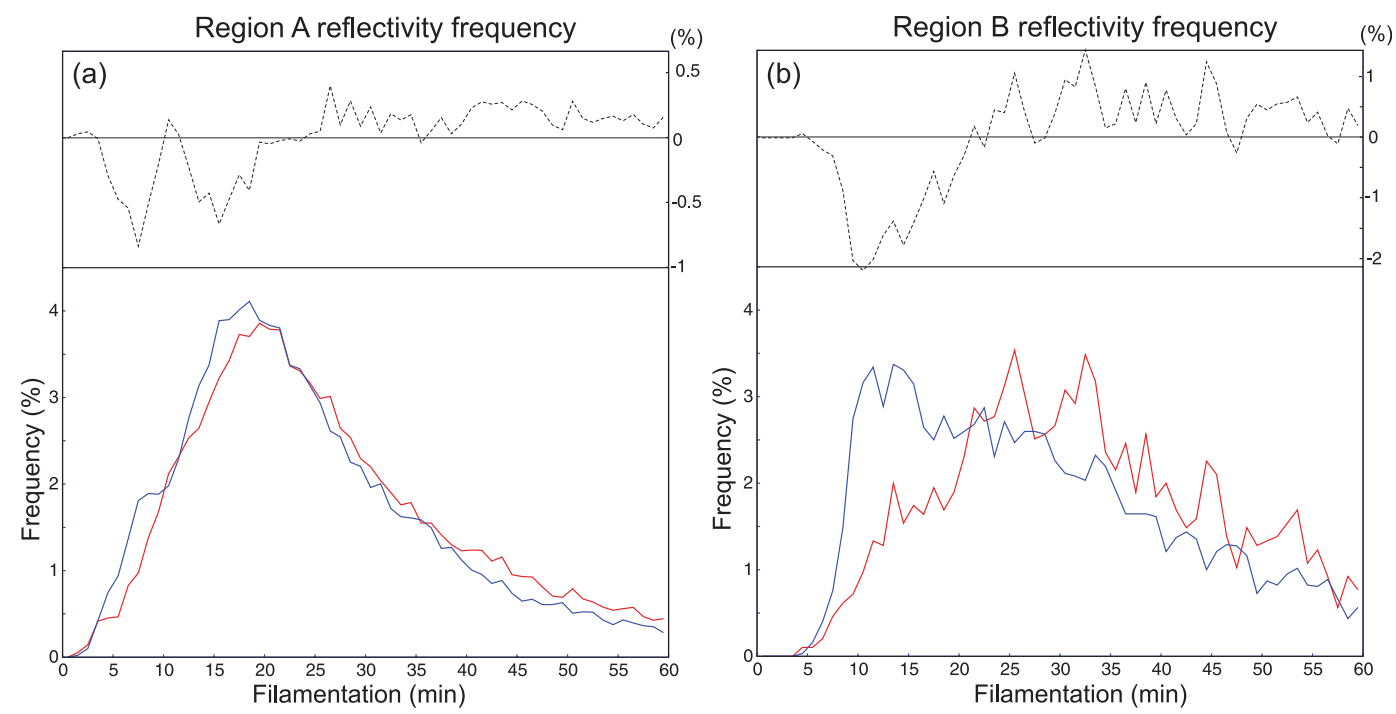

FIG. 8. The frequency distribution of filamentation time ( $\mathrm{min}$ ) for convective (red) and stratiform (blue) area for (a) region $\mathrm{A}$ and (b) region $\mathrm{B}$. The frequency is computed as the number of samples within 1 min divided by the total sample number of convective and stratiform echoes times 100 . The dashed line at top is the difference between the red and blue lines. The unit is the same as the bottom, but the scale is shown in the top-right portion of the frame.

be understood by considering the effects of vertical motion and filamentation on deep convection. Deep convection exists more frequently in regions of less filamentation effect ( $\tau_{\text {fil }}>24 \mathrm{~min}$ ), which is more affected by vertical motion, and tends to be suppressed by downward motion. On the other hand, the stratiform regime can either be suppressed by RF or by subsidence or both.

In the eyewall cloud (Fig. 9a), $\tau_{\text {fil }}<19$ min again delineates the regime where deep convection is suppressed. In the regime of upward motion there are slightly less convective and more stratiform echoes for $\tau_{\text {fil }}<$ $19 \mathrm{~min}$, and more convective and less stratiform echoes for $\tau_{\text {fil }}>19 \mathrm{~min}$. Because of the strong suppression effect of downward motion on deep convection, the convective regime occurs a bit less frequent than the stratiform regime. The differences are all much smaller than in the outer spiral band (region B) because convective forcing in the eyewall is much more intense and dominant.

\section{Summary}

The unique observations collected during TCS-08/TPARC made it possible to directly compute filamentation time in Typhoon Sinlaku, such that the effect of filamentation around the eyewall and the spiral band regions can be compared. Previous analyses of RF were based on strong TCs, many with concentric eyewalls. Our study connects the RF to a weak but intensifying TC.
A low-level RFZ is found during the reintensification stage of Typhoon Sinlaku 20-40 km from the TC center. Our analysis reveals that the RFZs are mostly collocated with the stratiform area, and its overlapping with deep convection areas is quite limited. There is a large variability of the reflectivity in the RFZ and the distribution suggests that the filamentation process suppresses deep convection in both regions. In the spiral band region $200 \mathrm{~km}$ from the TC center, the suppression is quite effective so that the convective regime occurs much less frequently than the stratiform regime for $\tau_{\text {fil }}<$ $24 \mathrm{~min}$. The situation is reversed for $\tau_{\text {fil }}>24 \mathrm{~min}$. The ratio of the deep convection occurrence over the stratiform precipitation varies from around $50 \%$ for $\tau_{\text {fil }}<$ $24 \mathrm{~min}$ to around $200 \%$ for $\tau_{\text {fil }}>24 \mathrm{~min}$. The collocation of the RFZ and the inner-edge stratiform area also suggests that the vortex-scale dynamics of strain effect may suppress convection on the inner side of the principal rainband. In the eyewall region with intense convective forcing the suppression is much smaller but still systematic and conspicuous, with the demarcation occurring at $\tau_{\text {fil }}=19 \mathrm{~min}$. In this case the ratio of the convective regime versus the stratiform regime occurrence differs by $\sim 10 \%$. Although this is a small magnitude, the fact that it shows up consistently with respect to a faster filamentation time (19 vs $24 \mathrm{~min}$ ) suggests that the filamentation dynamics play a role in the organization of deep convection in the eyewall. These effects are influenced by vertical motion that causes an 

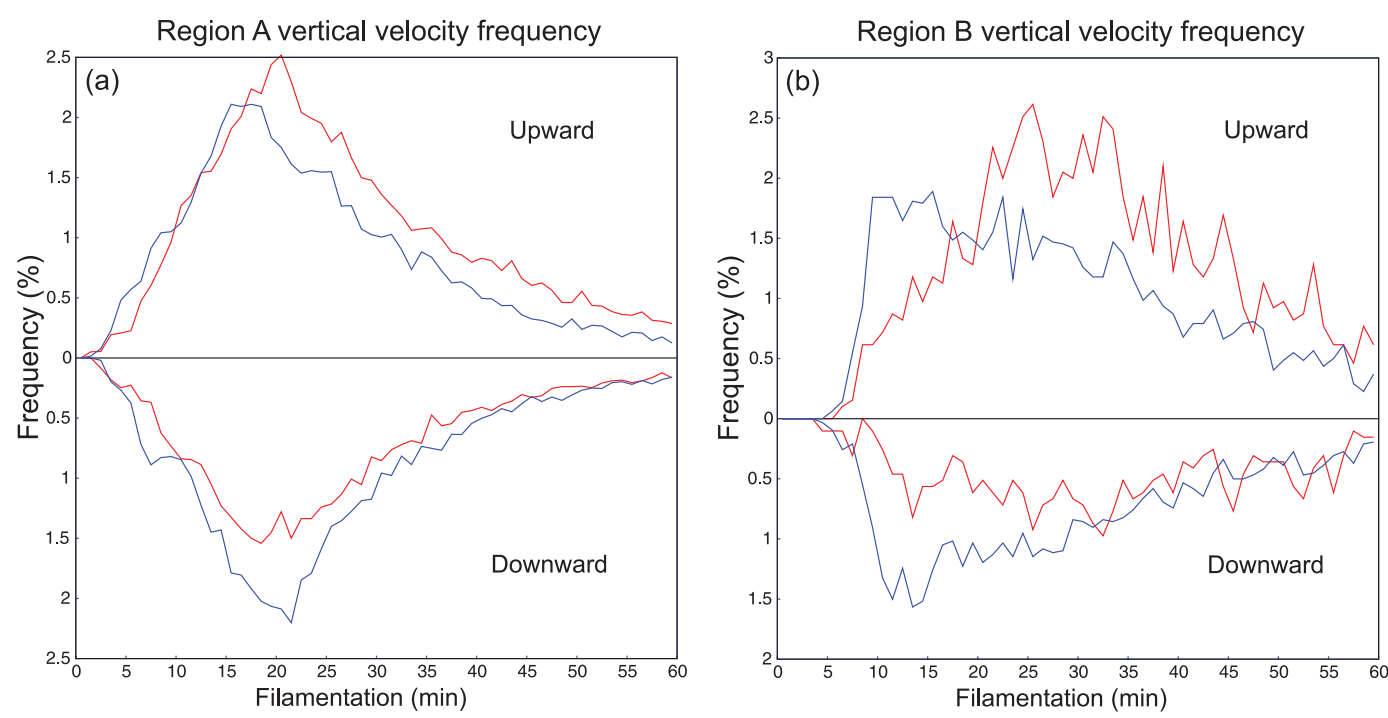

FIG. 9. The frequency distribution of filamentation time ( $\mathrm{min}$ ) for convective (red) and stratiform (blue) echoes in the (top) upward motion region and (bottom) downward motion region for (a) region A and (b) region B. The frequency is computed in the same manner as in Fig. 8.

asymmetric distribution of deep convection frequency between upward motion, which tends to help convection, and downward motion, which hinders convection. Probably because the stratiform area is a manifestation where convection is already hindered by filamentation or weak buoyancy or both, the effect of vertical motion is much less for stratiform precipitation, whose distribution remains nearly symmetric.

In closing, our results suggest that the vortex-scale filamentation dynamics may be important in suppressing deep convection and organizing spiral bands in the TC environment, which may affect the development and evolution of tropical cyclones. Since filamentation increases with increasing TC intensity, it may play a role in limiting convection particularly in the outer spiral cloud band region.

Acknowledgments. This research was supported by the U.S. Office of Naval Research to Naval Postgraduate School, under Awards N0001408WR20125, N0001409AF00002, and N0001410AF00002, and by Taiwan's National Research Council through Grants NSC96-2111-M-002-002, NSC97-2628-M-002-023, 97R006669, and MOTC-CWB-96-2M-01 to the National Taiwan University. HCK and CHL wish to thank ONR for supporting visits to the Naval Postgraduate School and for participation in the TCS-08 field experiment. CPC wishes to thank NSC for supporting visits to the National Taiwan University. We thank the entire NRL P-3 team for collecting and providing the data used in this research.

\section{REFERENCES}

Anagnostou, M. N., E. N. Anagnostou, and J. Vivekanandan, 2006: Correction for rain path specific and differential attenuation of X-band dual-polarization observations. IEEE Trans. Geosci. Remote Sens., 44, 2470-2480.

Biggerstaff, M. I., and S. A. Listemaa, 2000: An improved scheme for convective/stratiform echo classification using radar reflectivity. J. Appl. Meteor., 39, 2129-2150.

Cressman, G. P., 1959: An operational objective analysis system. Mon. Wea. Rev., 87, 367-374.

Didlake, A. C., and R. A. Houze, 2009: Convective-scale downdrafts in the principal rainband of Hurricane Katrina (2005). Mon. Wea. Rev., 137, 3269-3293.

Dritschel, D. G., and D. W. Waugh, 1992: Quantification of the inelastic interaction of unequal vortices in two-dimensional vortex dynamics. Phys. Fluids, 4A, 1737-1744.

Elsberry, R. L., and P. A. Harr, 2008: Tropical Cyclone Structure (TCS08) field experiment: Science basis, observational platforms, and strategy. Asia-Pac. J. Atmos. Sci., 44, 209-231.

Gamache, J. F., 1997: Evaluation of a fully-three dimensional variational Doppler analysis technique. Preprints, 28th Conf. on Radar Meteorology, Austin, TX, Amer. Meteor. Soc., 422-423.

Hack, J. J., and W. H. Schubert, 1986: Nonlinear response of atmospheric vortices to heating by organized cumulus convection. J. Atmos. Sci., 43, 1559-1573.

Hawkins, J. D., M. Helveston, T. F. Lee, F. J. Turk, K. Richardson, C. Sampson, J. Kent, and R. Wade, 2006: Tropical cyclone multiple eyewall configurations. Preprints, 27th Conf. on Hurricane and Tropical Meteorology, Monterey, CA, Amer. Meteor. Soc., 6B.1.[Available online at http://ams.confex.com/ ams/27Hurricanes/techprogram/paper_108864.htm.]

Hildebrand, P. H., and Coauthors, 1996: The ELDORA/ ASTRAIA airborne Doppler weather radar design and observations from TOGA COARE. Bull. Amer. Meteor. Soc., 77, 213-232. 
Jorgensen, D. P., 1984: Mesoscale and convective- scale characteristics of mature hurricanes. Part I: General observations by research aircraft. J. Atmos. Sci., 41, 1268-1286.

Kuo, H.-C., W. H. Schubert, C.-L. Tsai, and Y.-F. Kuo, 2008: Vortex interactions and barotropic aspects of concentric eyewall formation. Mon. Wea. Rev., 136, 5183-5198.

-, C.-P. Chang, Y.-T. Yang, and H.-J. Jiang, 2009: Western North Pacific typhoons with concentric eyewalls. Mon. Wea. Rev., 137, 3758-3770.

Leise, J. A., 1982: A multidimensional scale-telescoped filter and data extension package. NOAA Tech. Memo. ERL WPL-82, 19 pp. [Available from NOAA/ERL, 325 Broadway, Boulder, CO 80303.]

Matthaeus, W. H., and D. Montgomery, 1980: Selective decay hypothesis at high mechanical and magnetic Reynolds numbers. Ann. N.Y. Acad. Sci., 357, 203-222.

McWilliams, J. C., 1984: The emergence of isolated coherent vortices in turbulent flow. J. Fluid Mech., 146, 21-43.

Montgomery, M. T., and R. J. Kallenbach, 1997: A theory for vortex Rossby-waves and its application to spiral bands and intensity changes in hurricane. Quart. J. Roy. Meteor. Soc., 123, 435-465.
Rosenfeld, D., E. Amitai, and D. B. Wolff, 1995: Classification of rain regimes by the three-dimensional properties of reflectivity fields. J. Appl. Meteor., 34, 198-211.

Rozoff, C. M., W. H. Schubert, B. D. McNoldy, and J. P. Kossin, 2006: Rapid filamentation zones in intense tropical cyclones. J. Atmos. Sci., 63, 325-340.

,-- -, and J. Kossin, 2008: Some dynamical aspects of tropical cyclone concentric eyewalls. Quart. J. Roy. Meteor. Soc., 134, 583-593.

Steiner, M., R. A. Houze Jr., and S. E. Yuter, 1995: Climatological characterization of three-dimensional storm structure from operational radar and rain gauge data. J. Appl. Meteor., 34, 1978-2007.

Wakimoto, R. M., C.-H. Liu, and H. Cai, 1998: The Garden City, Kansas, storm during VORTEX 95. Part I: Overview of the storm's life cycle and mesocyclogenesis. Mon. Wea. Rev., 126, 372-392.

Wang, Y., 2008: Rapid filamentation zone in a numerically simulated tropical cyclone. J. Atmos. Sci., 65, 1158-1181.

Zhu, T., D.-L. Zhang, and F. Weng, 2004: Numerical simulation of Hurricane Bonnie (1998). Part I: Eyewall evolution and intensity changes. Mon. Wea. Rev., 132, 225-241. 